Planetary Systems in the Universe - Observation, Formation and Evolution

Proceedings IAU Symposium No. 202, (C)2004 IAU

Alan Penny, Pawel Artymowicz, Anne-Marie Lagrange, 8 Sara Russell, eds.

\title{
A stellar fly-by simulation giving $\beta$ Pic's disc asymmetries
}

\author{
John Larwood \\ Queen Mary \& Westfield College, Mile End Road, London E1 4NS, UK \\ Paul Kalas \\ University of California, 601 Campbell Hall, Berkeley, CA 94709, USA
}

\begin{abstract}
We have numerically investigated the dynamics of how a close stellar fly-by encounter of a symmetrical circumstellar planetesimal disc can give rise to the many kinds of asymmetries and substructures attributed to the dusty disc of $\beta$ Pic. We find three recognizable groupings of test particles that can be related to the morphology of the $\beta$ Pic disc. These are: highly eccentric and inclined orbit particles that reach apocentre in the southwest, moderately eccentric and inclined orbit particles that reach apocentre in the northeast, and a relatively unperturbed region inside $\sim 200 \mathrm{AU}$ radius.
\end{abstract}

\section{Introduction}

The starlight of $\beta$ Pic scatters off dust grains in its edge-on circumstellar disc (Smith \& Terrile 1984). Imaging reveals that the disc exhibits asymmetrical structure with respect to an ideal disc on projected radial scales from $\sim 50 \mathrm{AU}$ (Burrows et al. 1995; Mouillet et al. 1997) to 1000 AU (Smith \& Terrile 1987). Sensitive $R$-band images (Larwood \& Kalas 2001) show the northeast (NE) extension can be detected out to $1835 \mathrm{AU}$, whereas the southwest (SW) extension is detectable out to just $1450 \mathrm{AU}$, corresponding to a $\sim 25$ per cent length asymmetry. More recently, the longer NE midplane extension has been discovered to carry several brightness enhancements from $\sim 500$ to $800 \mathrm{AU}$ (Kalas et al. 2000), that have been attributed to a series of rings within the disc's midplane. Similar features in the SW extension were not detected and so eccentric rings were hypothesised. Kalas et al. (2000) presented a dynamical model in which the stellar fly-by scenario (Kalas \& Jewitt 1995) for generating the disc asymmetries was applied to the $\beta$ Pic system. Their finding was that the asymmetry types present in the $\beta$ Pic disc have analogues in the dynamical response of a particle disc to a perturbing stellar fly-by encounter. Furthermore, they demonstrated that these features could occur simultaneously with the formation of eccentric circumstellar rings.

As in the other works mentioned above we assume that the simulation particles' distribution represents the distribution of an underlying disc of planetesimals, which are parent bodies whose infrequent and therefore dynamically inconsequential collisions supply the dust scattering surface that is observed in the real system. A more complete model should include consideration of grain removal and generation processes in determining the appearance of the per- 
turbed disc. However, we defer treatment of those issues to future work, and focus here on the first-stage problem of the dynamics. This stage is important in deducing the mass and orbital parameters of the postulated stellar perturber, for which it is possible to perform star catalogue searches (Kalas, Deltorn \& Larwood 2001), and we proceed to do this by examining the length asymmetry, and other measures of the disc response, as a function of stellar encounter parameters.

\section{Results}

Larwood \& Kalas (2001) have described the origin of the transient circumstellar eccentric ring structures as being a general outcome of an encounter, due to the reflex motion of the primary star as the perturber passes through closest approach. The apparent ring system is actually an eccentric tightly-wound onearmed spiral pattern that gradually disappears owing to phase-mixing in the particles' orbital motion. Here we summarise time-invariant results found for the encounter parameters favoured by Kalas et al. (2000). Namely, a stellar perturber of mass $0.5 \mathrm{M}_{\odot}$, moving in a prograde parabolic trajectory with inclination of pericentre of $30^{\circ}$. Fig. 1 shows particle plots for some of the perturbed orbital elements in a disc of $10^{4}$ test particles that were set up in Keplerian circular coplanar orbits. The initial disc was set up between radii of 0.2 and 2 , and the distance of pericentre was 2.6. Kalas et al. (2000) suggested a scaling such that the unit of distance corresponds to $270 \mathrm{AU}$.

The disc response is separable into two major components corresponding to the direct tidal interaction between disc particles and the perturber, and the indirect interaction that results from the reflex motion of $\beta$ Pic (Larwood \& Kalas 2001). This is evident in Fig. 1 where we see, in the apocentre distance against longitude of pericentre plot, oppositely directed extensions that are asymmetric in length. The more diffuse extension is recognisable in the other panels of Fig. 1 , indicating that these particles have high eccentricities and inclinations, and small pericentre distances. This extension is formed by the direct interaction. The other extension, formed by the indirect interaction, has much lower eccentricity and inclination, and large pericentre distances. Respectively, we identify these extensions with the SW and NE extensions in the $\beta$ Pic disc.

\section{Conclusions}

The dynamical response of the models suggests that the $\beta$ Pic disc might be separable into different components corresponding to groupings of perturbed particle orbits. For example, we find that outwards of $\sim 250 \mathrm{AU}$ the SW extension may not have a distinct midplane owing to pumping of particle inclinations. The positions close to pericentre of the moderately eccentric NE midplane particles could account for the measurement of a SW midplane out to $\sim 650 \mathrm{AU}$, in superposition with the more diffuse vertically and radially extended component. When the optical image is vertically compressed (Larwood \& Kalas 2001) we are able to measure the SW extension to much larger radii (1450 AU). In our model the corresponding (very eccentric and inclined) particle orbits also intersect the disc near to their pericentres (down to $\sim 50 \mathrm{AU}$ ) possibly accounting for the in- 

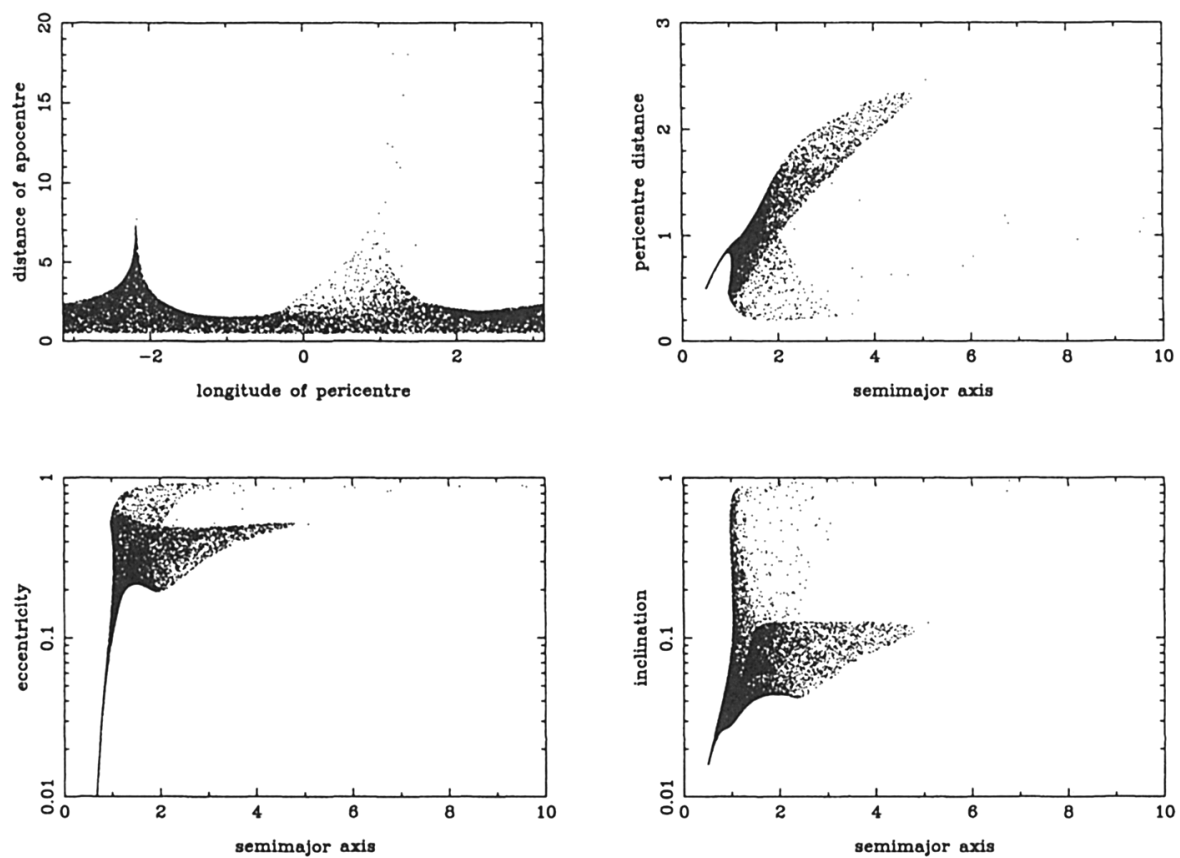

Figure 1. Orbital elements of perturbed disc particles.

ferred inner warp of the disc, which is aligned with the outer flared envelope in the SW. In summary, there are three recognizable particle groupings that can be related to the morphology of the $\beta$ Pic disc. These are: the highly eccentric and inclined particles that reach apocentre in the SW, the moderately eccentric and low inclination orbiting particles that reach apocentre in the NE, and the relatively unperturbed particles inside $\sim 200 \mathrm{AU}$ radius.

\section{References}

Burrows, C. J., Krist, J. E., \& Stapelfeldt, K. R. 1995, BAAS, 187, 32

Kalas, P., Deltorn, J. M., \& Larwood, J. 2001, ApJ, in press

Kalas, P., \& Jewitt, D. 1995, AJ, 110, 794

Kalas, P., Larwood, J., Smith, B. A., \& Shultz, A. 2000, ApJ, 530, L133

Larwood, J. D., \& Kalas, P. G. 2001, MNRAS, in press

Mouillet, D., Larwood, J. D., Papaloizou, J. C. B., \& Lagrange, A. M. 1997, MNRAS, 292,896

Smith, B. A., \& Terrile, R. J. 1984, Science, 226, 1421

Smith, B. A., \& Terrile, R. J. 1987, BAAS, 19, 829 